\title{
Gas-Phase Stability of Cluster Ions $\mathrm{SF}_{m}^{+}\left(\mathrm{SF}_{6}\right)_{n}$ with $m=0-5$ and $n=1-3$
}

\author{
Kenzo Hiraoka, Akitaka Shimizu, Akihito Minamitsu, Masayuki Nasu, \\ and Susumu Fujimaki \\ Faculty of Engineering, Yamanashi University, Takeda-4, Kofu 400, Japan
}

Shinichi Yamabe

Department of Chemistry, Nara University of Education, Takabatake-cho, Nara 630, Japan

\begin{abstract}
The gas-phase stabilities of cluster ions $\mathrm{SF}_{m}^{+}\left(\mathrm{SF}_{6}\right)_{n}$ with $m=0-5$ were determined by using a high pressure mass spectrometer. The bond energies of $\mathrm{SF}_{m}^{+}\left(\mathrm{SF}_{h}\right)_{1}$ were found to be less than $10 \mathrm{kcal} / \mathrm{mol}$ and to decrease with $m=0 \rightarrow 5$. There appear to be rather large gaps in the bond energies between $n=1$ and 2 for the clusters $\mathrm{SF}_{m}^{+}\left(\mathrm{SF}_{n}\right)_{n}$ with $m=0-4$. The structures of $\mathrm{SF}_{5}^{+}, \mathrm{SF}^{+}\left(\mathrm{SF}_{6}\right)_{1}, \mathrm{SF}_{3}^{+}\left(\mathrm{SF}_{6}\right)_{1}$, and $\mathrm{SF}_{5}^{+}\left(\mathrm{SF}_{6}\right)_{1}$ were investigated by ab initio molecular orbital calculations. For $\mathrm{SF}_{5}^{+}$, the $D_{3 h}$ geometry is found to be most stable and $C_{t i}$, is a transition state of the Berry pseudorotation. For the ion-molecule complexes, the "on-top hat" models were found to be the most stable structures. (J Am Soc Mass Spectrom 1995, 6, 1137-1142)
\end{abstract}

S ulfur hexafluoride has received attention for several decades because of its versatile practical applications. For example, it has been used widely as an efficient insulating gas for high voltage apparatus. Studies of negative ion formation via electron attachment to sulfur hexafluoride molecules in the gas phase have been extensive. Reactions of $\mathrm{SF}_{\mathrm{h}}$ with various positive ions also have been the subject of numerous investigations [1]. Creasey et al. [2] performed detailed measurements of the fragmentation channels and branching ratios for all of the valence states of $\mathrm{SF}_{6}^{+}$. Jiao and Freiser [1] studied reactions of transition-metal ions with $\mathrm{SF}_{6}$ in the gas phase. They pointed out that the inertness of $\mathrm{SF}_{6}$ is associated with the protective layer of $\mathrm{F}$ atoms that obstructs access to the reactive $S$ center by an attacking species.

The apparently conflicting results for the ionization energy of $\mathrm{SF}_{5}$ and the standard enthalpy of formation of $\mathrm{SF}_{5}^{+}$have been reported over the past 25 years [3]. Bohme and co-workers $[3,4]$ investigated ion-molecule reactions of $S_{2} F_{10}$ with various positive ions and suggested the existence of two isomers for $\mathrm{SF}_{5}^{+}$, that is, a ground-state trigonal bipyramidal $\left(D_{3 h}\right)$ structure and a less stable square pyramidal structure $\left(C_{4 i}\right)$. They found that the low energy $\left(D_{3 h}\right)$ isomer of $\mathrm{SF}_{5}^{+}$ reacts with $S_{2} F_{10}$ to produce $S_{2} F_{11}^{+}$and that the high

Address reprint requests to Kenzo Hiraoka, Faculty of Engineering, Yamanashi University, Takeda-4, Kofu 400, Japan. energy $\left(C_{+i}\right)$ isomer reacts with $S_{2} F_{10}$ to produce $\mathrm{SF}_{3}^{+}$. Cheung et al. [5] performed a theoretical investigation on the geometries of $\mathrm{SF}_{5}$ and $\mathrm{SF}_{5}^{+}$. They confirmed that the $\mathrm{SF}_{5}\left(C_{4 i}\right)$ and $\mathrm{SF}_{5}^{+}\left(D_{3 i}\right)$ structures are true minima, as reported in refs 3 and 4 . However, they found that $\mathrm{SF}_{5}^{+}\left(C_{4^{\prime}}\right)$ and $\mathrm{SF}_{5}\left(D_{3 / i}\right)$ structures have imaginary vibrational frequencies, which suggests that these structures are not at local minima. Thus the existence of two isomers for $\mathrm{SF}_{5}$ and $\mathrm{SF}_{5}^{+}$seems to be still controversial.

Protonated $\mathrm{SF}_{6}$ (i.e., $\mathrm{HSF}_{6}^{+}$) has not been considered to be stable against decomposition to $\mathrm{SF}_{5}^{+}$and $\mathrm{HF}[6$, 7]. Mackay et al. [8] first observed the production of $\mathrm{SF}_{6} \mathrm{H}^{+}$as a minor $(1 \%)$ channel in the reaction of $\mathrm{HCO}^{+}$with $\mathrm{SF}_{6}$. They predicted the proton affinity (PA) of $\mathrm{SF}_{6}$ to be $137 \pm 1 \mathrm{kcal} / \mathrm{mol}$. Latimer and Smith [9] studied reactions that take place within the core of a free jet flow. They observed the stable $\mathrm{HSF}_{6}^{+}$near 0 $\mathrm{K}$ and determined the proton affinity of $\mathrm{SF}_{6}$ to be $137.9 \pm 2.0 \mathrm{kcal} / \mathrm{mol}$, which is in good agreement with the value reported by Mackay et al. [8].

In this work, the clustering reactions of $\mathrm{SF}_{m}^{+}(m=$ $0-5)$ with $\mathrm{SF}_{6}$ were measured via a high pressure mass spectrometer. It was confirmed that $\mathrm{SF}_{6}$ is a poor nucleophilic reagent toward $\mathrm{SF}_{m \prime}^{+}(m=0-5)$ ions and that the interaction between $\mathrm{SF}_{5}^{+}$and $\mathrm{SF}_{6}$ is exceptionally weak compared to other $\mathrm{SF}_{m}^{+}$ions. Theoretical calculations on the structures of $\mathrm{SF}^{+}, \mathrm{SF}_{3}^{+}, \mathrm{SF}_{5}^{+}$, and their clusters with $\mathrm{SF}_{6}$ also were performed. 


\section{Experimental and Computational}

The experiments were performed with a pulsed-electron-beam high pressure mass spectrometer [10,11]. A copper ion source equipped with a cryocooler (Iwatani Plantech, type-S030) was used to perform low temperature measurements. The $\mathrm{N}_{2}$ carrier gas was purified by passing it through a liquid $\mathrm{N}_{2}$ cooled 5-A molecular sieve trap at 3 torr. $\mathrm{SF}_{6}$ (Iwatani, reagent grade) was introduced into 3 torr of $\mathrm{N}_{2}$ carrier gas through a flow-controling stainless steel capillary $(0.1 \mathrm{~mm} \times 1$ $\mathrm{m})$. The pressure of $\mathrm{SF}_{6}$ introduced into the $\mathrm{N}_{2}$ buffer gas was $0.03-0.8$ torr dependent on the experimental conditions. The surface of the ion source was coated with colloidal graphite to prevent charging of the ion source.

The reagent gas in the reaction chamber was ionized by a pulsed 2-keV electron beam. The produced ions were sampled through a slit made of razor blades (10 $\mu \mathrm{m} \times 1 \mathrm{~mm}$ ) and were mass-analyzed by a quadrupole mass spectrometer (ULVAC, MSQ-400). The ions were collected in a multichannel analyzer as a function of arrival time after the electron pulse.

Geometries and electronic distributions of $\mathrm{SF}^{+}, \mathrm{SF}_{3}^{+}$, $\mathrm{SF}_{5}^{+}$, and their $\mathrm{SF}_{6}$ bound clusters were determined with ab initio molecular orbital calculations. The basis set used is $6-31 \mathrm{G}^{(*)}$, where the polarization function $\left({ }^{*}\right)$ is set on the sulfur atom. This set is needed to describe the hypervalent bond in $\mathrm{SF}_{6}$. RHF/6-31G(*) calculations were carried out for $\mathrm{SF}_{3}^{+}, \mathrm{SF}_{5}^{+}$, and their clusters. The UHF $/ 6-31 \mathrm{G}^{(*)}$ calculation was used for $\mathrm{SF}^{+}$and $\mathrm{SF}^{+}\left(\mathrm{SF}_{6}\right)_{1}$, because these species are triplet spin states. Spin contaminations were found to be safely small $\left(\left\langle S^{2}\right\rangle=2.02\right)$. For the controversial geometries of $\mathrm{SF}_{5}^{+}$, the following additional calculation was carried out. First, the MP2/6-31G* (used also in [5]) full geometry optimization was made without symmetry constraint. In [4] and [5], $C_{4}$, and $D_{3 / h}$ geometries were assumed and the interrelationship between them still was unclear. Second, at the practical $\mathrm{C}_{4}$, geometry, MP2/6-31G* vibrational analysis and subsequent trace of the intrinsic reaction coordinate (IRC) [12] were performed. This IRC calculation gave the overall potential surface of the $\mathrm{SF}_{5}^{+}$isomerization for the first time. All calculations were performed with Gaussian 92 [13] installed on the CONVEX-220 computer (Information Processing Center at Nara University of Education).

\section{Experimental Results}

When the reagent gas $\left(\mathrm{N}_{2}\right.$ buffer gas that contains a small amount of $\mathrm{SF}_{6}$ ) was ionized by a 2-keV electron beam, the positive ions $\mathrm{SF}_{m}^{+}$with $m=0-5$ were observed as major ions. The relative intensities of $\mathrm{S}^{+}$, $\mathrm{SF}^{+}, \mathrm{SF}_{2}^{+}, \mathrm{SF}_{3}^{+}, \mathrm{SF}_{4}^{+}$, and $\mathrm{SF}_{5}^{+}$were $0.1,0.3,0.4,2.8,1.0$, and 95.4, respectively, under typical experimental conditions. The $\mathrm{SF}_{6}^{+}$ion was not detected. The absence of $\mathrm{SF}_{6}^{+}$is due to the repulsive nature of the ground state
$\mathrm{SF}_{6}^{+}$, which fragments on a subpicosecond time scale by loss of a fluorine atom to $\mathrm{SF}_{5}^{+}$and $\mathrm{F}$ [4]. Under the present experimental conditions, the decay rates of $\mathrm{SF}_{m}^{+}$ions with $m=0-5$ were found to be slow enough to measure the equilibria for reaction 1 :

$$
\mathrm{SF}_{m}^{+}\left(\mathrm{SF}_{6}\right)_{n-1}+\mathrm{SF}_{6}=\mathrm{SF}_{m}^{+}\left(\mathrm{SF}_{6}\right)_{n} \quad(m=0-5)
$$

As an example, the van't Hoff plots for reactions $S_{m}^{+}$ $\left(\mathrm{SF}_{6}\right)_{n-1}+\mathrm{SF}_{6}=\mathrm{S}_{m}^{+}\left(\mathrm{SF}_{6}\right)_{n}(m=0,5)$ are shown in Figure 1 . Thermochemical data for reaction 1 with $m=$ 0-5 obtained from the measured van't Hoff plots are summarized in Table 1. In Figure 2, the $-\Delta H_{n-1, n}^{\circ}$ values for reaction 1 are shown as a function of $n$.

As shown in Figure 2, the measured $-\Delta H_{n-1, n}^{\circ}$ values are less than $10 \mathrm{kcal} / \mathrm{mol}$. This indicates that the $\mathrm{SF}_{6}$ molecule is a rather weak nucleophilic reagent as was pointed out by Rauth et al. [14]. In Figure 2, the $-\Delta H_{0,1}^{\circ}$ values decrease in the order of $m=0 \rightarrow 5$. The $-\Delta H_{0,1}^{\circ}$ values for $m=0,1$ and $m=2,3,4$ are close to each other whereas the $-\Delta H_{0,1}^{\circ}$ value with $m=5$ is much smaller. This suggests that the bonding in the clusters $\mathrm{SF}_{m}^{+}\left(\mathrm{SF}_{6}\right)_{1}$ with $m=0,1$ and $m=2-4$ is similar. The smallest bond energy for $\mathrm{SF}_{5}^{+}\left(\mathrm{SF}_{6}\right)_{1}$ may be due to the well-dispersed positive charge in the $\mathrm{SF}_{5}^{+}$ cation with a trigonal bipyramidal structure.

In Figure 2, a sudden drop appears in $-\Delta H^{\circ},-1, n$ between $n=1$ and 2 for $m=0-4$. This $n$ dependence indicates that the $n=1$ clusters are relatively more stable than the $n=2$ clusters for $\mathrm{SF}_{n}^{+}\left(\mathrm{SF}_{6}\right)_{n}$ with $m=0-4$. This may suggest a slight participation of a
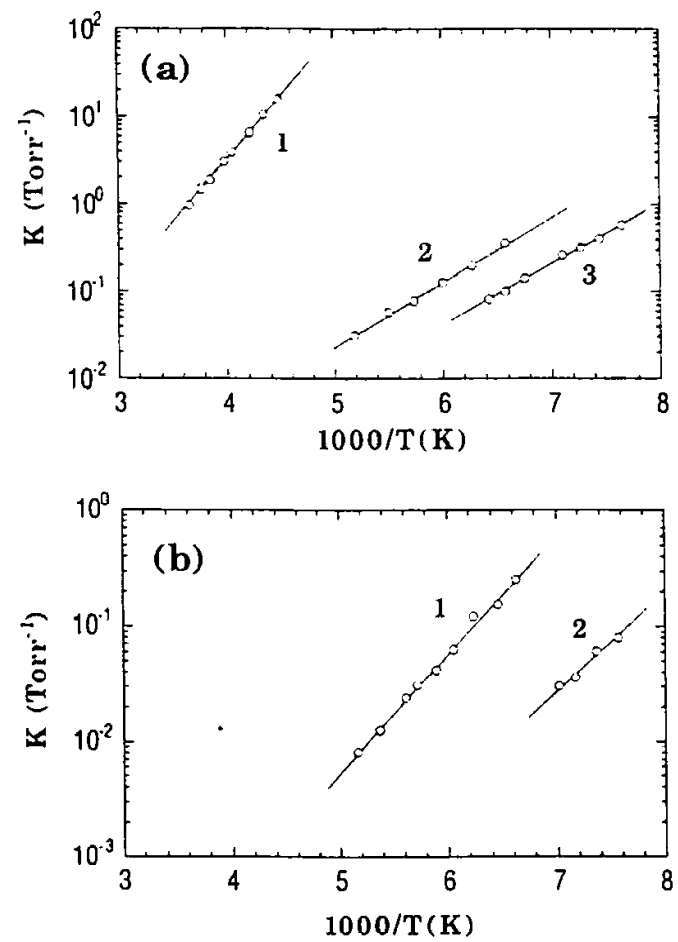

Figure 1. van't Hoff plots of the gas-phase clustering reaction, (a) $\mathrm{S}^{+}\left(\mathrm{SF}_{6}\right)_{n-1}+\mathrm{SF}_{6}=\mathrm{S}^{+}\left(\mathrm{SF}_{6}\right)_{n}$ and (b) $\mathrm{SF}_{5}^{+}\left(\mathrm{SF}_{6}\right)_{n-1}+\mathrm{SF}_{5}=$ $\mathrm{SF}_{5}^{+}\left(\mathrm{SF}_{6}\right)_{1}$. Integer numbers in the figure represent values of $\|$. 
Table 1. Experimental thermochemical data, $\Delta H_{n-1, n}^{\circ}(\mathrm{kcal} / \mathrm{mol})$ and $\Delta S^{\circ}{ }_{n-1, n}(\mathrm{cal} / \mathrm{mol} \mathrm{K})$ (standard state, $\left.1 \mathrm{~atm}\right)$, for gas-phase clustering reactions, $\mathrm{SF}_{m}^{+}\left(\mathrm{SF}_{6}\right)_{n-1}+\mathrm{SF}_{6}=\mathrm{SF}_{m}^{+}\left(\mathrm{SF}_{6}\right)_{n}$ for $m=0-5^{a}$

\begin{tabular}{|c|c|c|c|c|c|c|c|c|c|c|c|c|}
\hline \multirow[b]{2}{*}{$n-1, n$} & \multicolumn{2}{|c|}{$\mathrm{S}^{+}\left(\mathrm{SF}_{6}\right)_{n}-1, n$} & \multicolumn{2}{|c|}{$\mathrm{SF}^{+}\left(\mathrm{SF}_{6}\right)_{n}-1, n$} & \multicolumn{2}{|c|}{$\mathrm{SF}_{2}^{+}\left(\mathrm{SF}_{6}\right)_{n}-1, n$} & \multicolumn{2}{|c|}{$\mathrm{SF}_{3}^{+}\left(\mathrm{SF}_{6}\right)_{n}-1, n$} & \multicolumn{2}{|c|}{$\mathrm{SF}_{4}^{+}\left(\mathrm{SF}_{6}\right)_{n}-1, n$} & \multicolumn{2}{|c|}{$\mathrm{SF}_{5}^{+}\left(\mathrm{SF}_{6}\right)_{n}-1, n$} \\
\hline & $-\Delta H^{\circ}$ & $-\Delta S^{\circ}$ & $-\Delta H^{\circ}$ & $-\Delta S^{\circ}$ & $-\Delta H^{\circ}$ & $-\Delta S^{\circ}$ & $-\Delta H^{\circ}$ & $-\Delta S^{\circ}$ & $-\Delta H^{\circ}$ & $-\Delta S^{\circ}$ & $-\Delta H^{\circ}$ & $-\Delta S^{\circ}$ \\
\hline 0,1 & 9.4 & 21 & 9.1 & 20 & 7.8 & 18 & 7.4 & 25 & 7.2 & 23 & 4.7 & 21 \\
\hline & & & {$[7.07]^{c}$} & & & & {$[7.63]^{\mathrm{c}}$} & & & & {$[5.49]^{c}$} & \\
\hline 1,2 & 3.4 & 11 & 3.8 & 16 & 5.0 & 22 & 4.5 & 19 & 4.6 & 14 & 3.6 & 19 \\
\hline 2.3 & 3.0 & 11 & 3.7 & 17 & 4.2 & 20 & 4.0 & $(19)^{\mathrm{b}}$ & & & & \\
\hline
\end{tabular}

${ }^{\circ}$ Experimental errors for $\Delta H_{0.1}^{\circ}$ and $\Delta S_{0.1}^{\circ}$ are $0.5 \mathrm{kcal} / \mathrm{mol}$ and $2 \mathrm{cal} / \mathrm{mol} \mathrm{K}$, and those for $\Delta H_{n}^{\circ}-1, n$ and $\Delta S_{n}^{\circ}-1, n$ with $n \geq 2$ are $0.2 \mathrm{kcal} / \mathrm{mol}$ and $2 \mathrm{cal} / \mathrm{mol} \mathrm{K}$, respectively.

Entropy value assumed.

"Values in square brackets denote MP2/6-31 G*//HF/6-31 $\mathrm{G}^{*}$ theoretical binding energies, $\Delta E s$.

covalent bond in these $n=1$ clusters. However, the ground electronic spin states of $\mathrm{S}^{+}$and $\mathrm{SF}^{+}$are quartet and triplet, respectively, and the spin conservation in the formation of cluster ions $(n=1)$ with some covalency may be highly endothermic for these cations. Thus, the observed sudden drop in the $-\Delta H^{\circ}{ }_{n-1, n}$ between $n=1 \rightarrow 2$ for $\mathrm{SF}_{m}^{+}\left(\mathrm{SF}_{6}\right)_{n}(m=0-4)$ may be due to mainly the steric effect (e.g., exchange repulsion between ligand $\mathrm{SF}_{6}$ molecules) in the cluster ions.

In Table 1, a drastic decrease in $-\Delta S_{n-1, n}^{\circ}$ is observed with $n=1 \rightarrow 2$ for $\mathrm{S}^{+}\left(\mathrm{SF}_{6}\right)_{n}$. This indicates that the freedom of motion for $n \geq 2 \mathrm{SF}_{6}$ ligands is much less restricted than the $n=1 \mathrm{SF}_{6}$. It is likely that the $\mathrm{S}^{+}\left(\mathrm{SF}_{6}\right)_{1}$ forms the core in the larger cluster ions, that is, $\left[\mathrm{S}^{+}\left(\mathrm{SF}_{6}\right)_{1}\right]\left(\mathrm{SF}_{6}\right)_{n-1}$.

\section{Computational Results}

In the previous section, three groups of the binding energies ( $m=0,1, m=2,3,4$, and $m=5$ ) have been recognized. Geometries and electronic distributions of $n t=1,3,5$ are considered in respective groups. This selection comes from the fact that $\mathrm{S}^{+}\left(\mathrm{SF}_{6}\right)_{1}, \mathrm{SF}_{2}^{+}\left(\mathrm{SF}_{6}\right)_{1}$, and $\mathrm{SF}_{4}^{+}\left(\mathrm{SF}_{6}\right)_{1}$ require a more accurate (multiconfigurational) wavefunction than the Hartree-Fock and their geometry optimization is beyond the present computational facility.

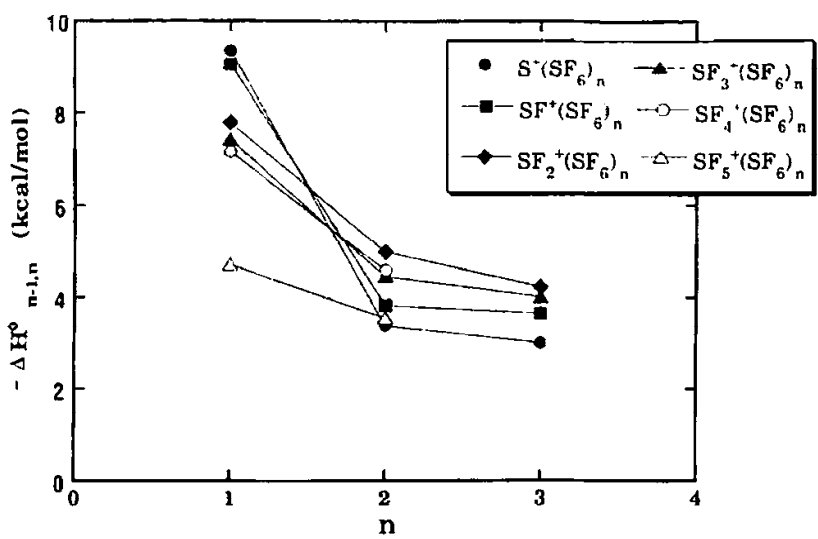

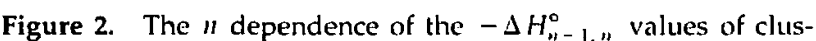
tering reaction $1, \mathrm{SF}_{m}^{+}\left(\mathrm{SF}_{6}\right)_{n-1}+\mathrm{SF}_{6}=\mathrm{SF}_{m}^{+}\left(\mathrm{SF}_{6}\right)_{n}$ with $m=0-5$.
Figure 3 shows fragments $\mathrm{SF}_{6}, \mathrm{SF}^{+}$, and $\mathrm{SF}_{3}^{+}$before cluster formation. The $\mathrm{SF}_{6}$ molecule is confirmed to be of $O_{h}$ point group, and the $S$ atom is quite cationic $(+1.93) . \mathrm{SF}^{+}$is valence isoelectronic with $\mathrm{S}_{2}$ and $\mathrm{O}_{2}$, and it is a triplet spin state. The sulfur atom in $\mathrm{SF}^{+}$is quite cationic $(+1.20) . \mathrm{SF}_{3}^{+}$is a nonplanar molecule as is expected from the Walsh rule [15] for $A B_{3}$-type molecules. Although the sulfur atom in $\mathrm{SF}_{3}^{+}$is very cationic $(+1.58)$, there is a lone-pair orbital along the principal axis.

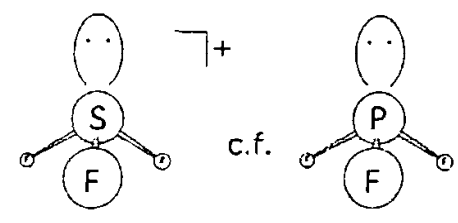

Therefore, the principal-axis coordination is thought to be unfavorable due to exchange repulsion.

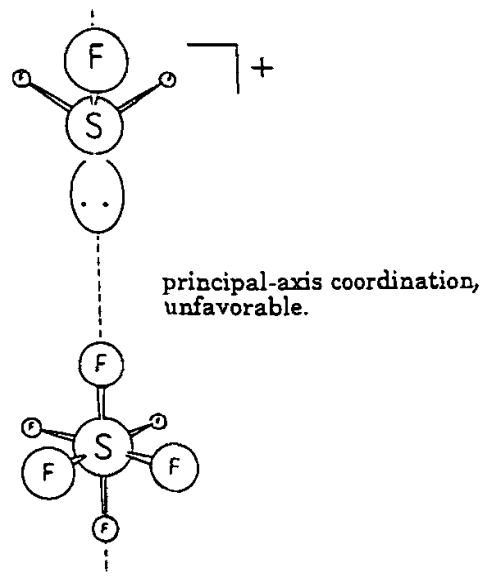

Figure 4 shows geometries for $\mathrm{SF}_{5}^{+}$along the isomerization path. The $C_{4 v}$, geometry is confirmed to be a transition state [5] with the sole imaginary frequency $\nu=140.1 \mathrm{i} \mathrm{cm}^{-1}$. Next, the reaction-coordinate vector that corresponds to the frequency is traced along the reaction path (IRC) [12]. Two energy-minimum points $(S=-5.5$ and +5.5$)$ are found to be $D_{3 h}$ isomers. Thus, the $C_{4 v}$ geometry is of a transition state for the Berry pseudorotation [16] between two $D_{3 h}$ geome- 


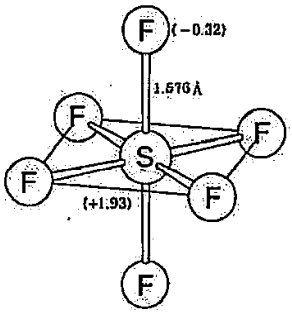

$\mathrm{Oh}$

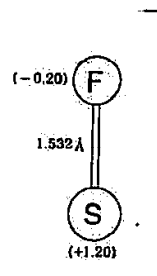

Coin.

(triplet)

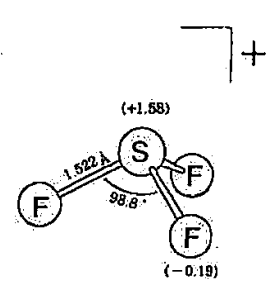

C3y

Figure 3. Geometries of $\mathrm{SF}_{6}, \mathrm{SF}^{+}$, and $\mathrm{SF}_{3}^{+}$fully optimized with $\left.\mathrm{RHF} / 6-31 \mathrm{G}^{*}\right)$. Values in parentheses denote atomic charges (positive, cationic).

tries. A similar pseudorotation has been found in $\mathrm{SiF}_{4}^{-}$ [17], which is isoelectronic with $\mathrm{SF}_{5}^{+}$. Thus, the pseudorotation is a common characteristic in $\mathrm{SiX}_{5}^{-}, \mathrm{PX}_{5}$, and $\mathrm{SX}_{5}^{+}(\mathrm{X}=\mathrm{F}$ and $\mathrm{Cl})$.

The $\mathrm{C}_{4 v} \mathrm{SF}_{5}^{+}$might give an effective Coulombic attraction along the principal axis.

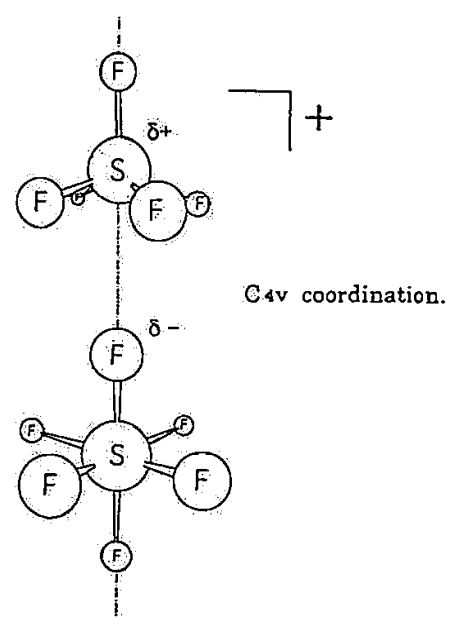

Berry Pseudorotation of $\mathrm{SF}_{5}{ }^{+}$

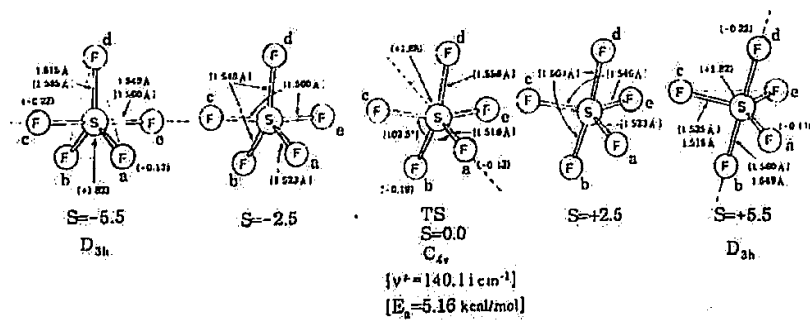

Figure 4. Interrelationship between $C_{4 v}$ and $D_{3 h}$ geometries of $\mathrm{SF}_{5}^{+}$along the intrinsic reaction coordinate (IRC) [12] of the isomerization. From the transition state (TS, the $C_{4 v}$ symmetry), two ways of the distortion lead to $D_{3 h}$ stable isomers. Bracket values are of $M P 2 / 6-31 G^{*}$ for the IRC calculation. Interrupted lines stand for principal axes of $C_{4 u}$ and $D_{34}$ point groups. $S$ values denote reaction coordinates (Bohr $\mathrm{u}^{1 / 2}$ ), $\nu$ and $E_{n}$ of $C_{40}$ are the sole imaginary frequency and the activation energy of $\mathrm{MP} 2 / 6-31 \mathrm{G}^{*}$, respectively.

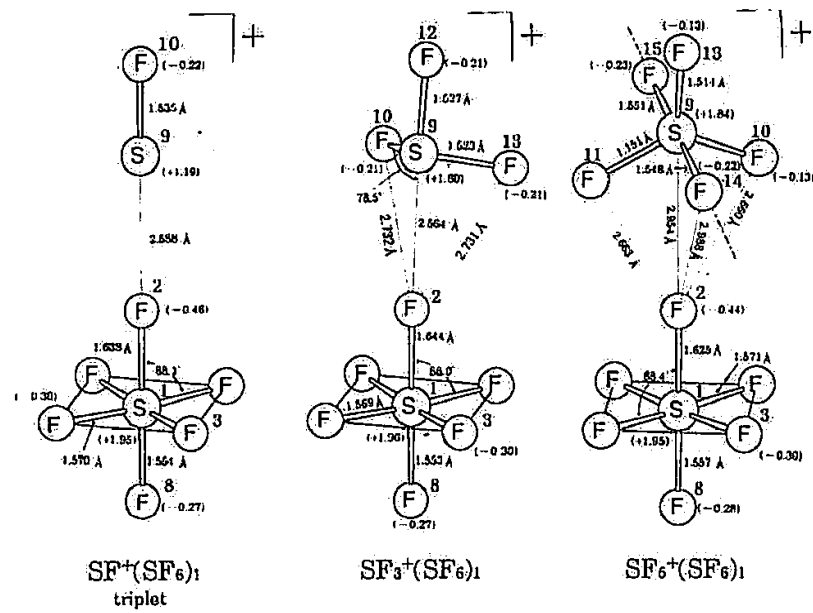

Figure 5. Geometries of three $n=1$ clusters. For $\mathrm{SF}_{5}^{+}\left(\mathrm{SF}_{6}\right)_{1}$, the principal axis of the $\mathrm{SF}_{5}^{+}$moiety is shown by an interrupted line

It is of structural interest to examine whether the $C_{4 \mathrm{v}}$ coordination is favored in spite of the $E_{\mathrm{a}}=5.16$ $\mathrm{kcal} / \mathrm{mol}$ instability shown in Figure 4.

Figure 5 exhibits optimized structures of three $n=1$ clusters. As shown in the figure, the top-hat coordinations are found to be most stable. No chelate-bond-type coordinations are found.

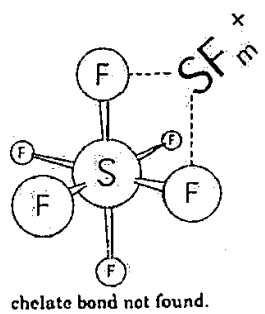

In $\mathrm{SF}^{+}\left(\mathrm{SF}_{6}\right)_{1}$, the intermolecular $\mathrm{S}^{9} \ldots-\mathrm{F}^{2}$ distance is large $(=2.59 \mathrm{~A})$, which corresponds to the small $-\Delta H_{0,1}^{\circ}=9.1 \mathrm{kcal} / \mathrm{mol}$ in Table 1 . The $\mathrm{S}-\mathrm{F}$ bond length $(=1.535 \mathrm{~A})$ in the $\mathrm{SF}^{+}$moiety is almost the same as that in the free $\mathrm{SF}^{+}(=1.532 \mathrm{~A}$ in Figure 3). On the other hand, the $\mathrm{SF}_{6}$ geometry is somewhat distorted due to cluster formation. Noticeably, the $S^{1}-$ $\mathrm{F}^{2}$ bond is elongated $\left(1.576 \mathrm{~A}\right.$ in $\mathrm{SF}_{6} \rightarrow 1.638 \mathrm{~A}$ in the cluster). Along with this elongation, the $F^{2}$ atom becomes more anionic $(-0.46)$ than that $(-0.32)$ in the free $\mathrm{SF}_{6}$. Because the $\mathrm{SF}_{6} \rightarrow \mathrm{SF}^{+}$charge shift is small $\left(0.03 e\right.$ in $\mathrm{SF}_{6} \rightarrow \mathrm{SF}^{+}$and $\mathrm{SF}_{6} \rightarrow \mathrm{SF}_{3}^{+}$, and $0.02 e$ in $\mathrm{SF}_{6}$ $\rightarrow \mathrm{SF}_{5}^{+}$; present work), the $\mathrm{SF}_{6}$ distortion is ascribed to the polarization.

$\mathrm{SF}_{3}^{+}\left(\mathrm{SF}_{6}\right)_{1}$ has the structure of the $S_{N} 2$ back-side attack along a $\mathrm{S}-\mathrm{F}$ bond in $\mathrm{SF}_{3}^{+}$. The principal-axis coordination is not obtained as is predicted. $\mathrm{F}^{2} \ldots . \mathrm{F}^{10}$ 
and $F^{2}--F^{13}$ distances are 2.73 A. Exchange repulsion of these pairs blocks the further $S^{9} \cdots-F^{2}$ approach. The geometry of $\mathrm{SF}_{6}$ is distorted slightly as in $\mathrm{SF}^{+}\left(\mathrm{SF}_{6}\right)_{1}$.

In $\mathrm{SF}_{5}^{+}\left(\mathrm{SF}_{6}\right)_{1}$, the $D_{3 h} \mathrm{SF}_{5}^{+}$is retained and is not isomerized to the $\mathrm{C}_{4 \mathrm{v}}$, structure, although the latter is a better electrophile toward $\mathrm{SF}_{6} . \mathrm{F}^{10}, \mathrm{~F}^{11}$, and $\mathrm{F}^{14}$ in $\mathrm{SF}_{5}^{+}$ block the $S^{9} \cdots-F^{2}$ attraction, which leads to its large distance $(=2.95 \mathrm{~A})$ and consequently to the small binding energy $(=4.7 \mathrm{kcal} / \mathrm{mol}$ in Table 1$)$.

The geometries of the $\mathrm{SF}_{6}$ adducts with $n=2$ may be estimated in view of the three $n=1$ geometries shown in Figure 5. In $\mathrm{SF}^{+}\left(\mathrm{SF}_{6}\right)_{1}$, the principal-axis coordination has been obtained. The second $\mathrm{SF}_{6}$ is obligated to be linked with $\mathrm{SF}^{+}$as shown in succeeding text. This drastic change of the sterically crowded coordination corresponds to the large fall-off of $-\Delta H^{\circ}{ }_{n-1, n}(9.1 \mathrm{kcal} / \mathrm{mol}$ of $n=1 \rightarrow 3.8 \mathrm{kcal} / \mathrm{mol}$ of $n=2)$ in Table 1 . In $\mathrm{SF}_{3}^{+}\left(\mathrm{SF}_{6}\right)_{2}$, the second $\mathrm{SF}_{6}$ is directed to another $S-F$ (say, $S^{9}-F^{13}$ ) bond. In $\mathrm{SF}_{5}^{+} \quad\left(\mathrm{SF}_{6}\right)_{2}$, the second $\mathrm{SF}_{6}$ is attached to the $\left(\mathrm{F}^{13}, \mathrm{~F}^{15}, \mathrm{~F}^{11}\right)$ or $\left(\mathrm{F}^{13}, \mathrm{~F}^{15}, \mathrm{~F}^{10}\right)$ triangle of $\mathrm{SF}_{5}^{+}$.

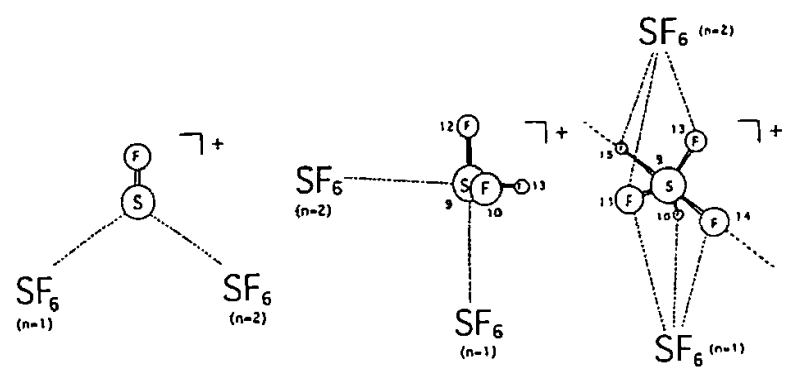

The smallest fall-off in $-\Delta H_{n-1, n}^{\circ}(4.7 \mathrm{kcal} / \mathrm{mol}$ for $n=1 \rightarrow 3.5 \mathrm{kcal} / \mathrm{mol}$ for $n=2$ ) has been obtained for $\mathrm{SF}_{5}^{+}\left(\mathrm{SF}_{6}\right)_{n}$ in Table 1. This comes from the almost undisturbed $D_{3 h} \mathrm{SF}_{5}^{+}$, where the coordination of $\mathrm{SF}_{6}$ ligands to the cation center (the sulfur atom) is blocked by two equatorial and one apical fluorine atoms. As an indication of structural analyses, $n=1$ binding energies are controled by the extent of the F.-.-F exchange repulsion. The bonding is mainly electrostatic with slight participation of a covalent bond (i.e., the bonding density 0.02 between $F^{2}$ and $S^{9}$ atoms in three clusters). The $n=1 \rightarrow 2$ fall-off in energies is determined by the extent of $\mathrm{SF}_{6} \cdots \mathrm{SF}_{6}$ repulsion. The computed binding energies ( $\Delta E \mathrm{~s}$ ) given in square brackets in Table 1 for $\mathrm{SF}_{3}^{+}\left(\mathrm{SF}_{6}\right)_{1}$ and $\mathrm{SF}_{5}^{+}\left(\mathrm{SF}_{6}\right)_{1}$ are in good agreement with $\Delta H^{\circ}{ }_{0,1} \mathrm{~s}$. However, $-\Delta E$ of $\mathrm{SF}^{+}\left(\mathrm{SF}_{6}\right)_{1}$ $(7.07 \mathrm{kcal} / \mathrm{mol})$ is underestimated. The UHF triplet calculation has some problems.

Although $\mathrm{SF}_{m}^{+}\left(\mathrm{SF}_{6}\right)_{1}$ clusters $(m=0,2,4)$ have not been dealt with theoretically, their geometries can be predicted. $\mathrm{S}^{+}$--- $\mathrm{SF}_{6}$ would be of principal-axis coordination with $\mathrm{C}_{4 v}$, symmetry. $\mathrm{SF}_{2}^{+}$is a $\pi$ radical, and $\mathrm{SF}_{4}^{+}$is composed of $\mathrm{SF}_{2}^{+}$and two fluorine atoms attached perpendicularly to the radical center $\mathrm{S}^{+}$.
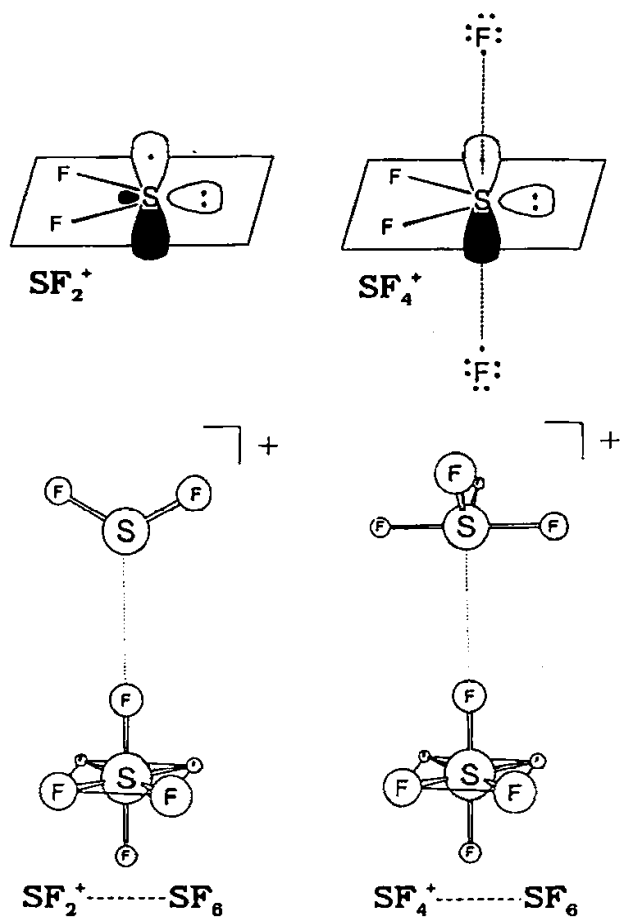

$\mathrm{SF}_{2}^{+}$and $\mathrm{SF}_{4}^{+}$would be coordinated by the electrostatic force to $\mathrm{SF}_{6}$ along the $\mathrm{SF}_{6}$ principal axis with $C_{2 i}$ symmetry. The $\mathrm{SF}_{2}^{+} \cdots \mathrm{SF}_{6}$ geometry is based on our rough calculations. Although the $\mathrm{SF}_{4}^{+} \cdots \mathrm{SF}_{6} \mathrm{C}_{2}$, geometry is reasonable, the charge-transfer $\pi$ radical on-top coordination in $\mathrm{SF}_{2}^{+} \ldots \mathrm{SF}_{6}$ also might be likely. In any event, the principal-axis coordination of the sulfur atom in $\mathrm{SF}_{m}^{+}$is a uniform pattern in $\mathrm{SF}_{m}^{+}-\mathrm{-mF}_{6}$ geometries.

\section{Concluding Remarks}

In this work, clustering reactions between sulfur hexafluoride and its fragment cations have been investigated. All $\mathrm{SF}_{m}^{+}\left(\mathrm{SF}_{6}\right)_{1}$ clusters have small binding energies $(<10 \mathrm{kcal} / \mathrm{mol})$ and they involve top-hat coordinations. The charge transfer $\mathrm{SF}_{6} \rightarrow \mathrm{SF}_{m}^{+}$is small, but the polarization in $\mathrm{SF}_{6}$ is appreciable. The controversial geometries of $\mathrm{SF}_{5}^{+}$have been examined, and the Berry pseudorotation of $C_{4 i}$ (TS) $\rightarrow D_{3 h}$ isomers has been obtained along the intrinsic reaction coordinate. The $D_{3 h}$ geometry is almost retained in $\mathrm{SF}_{5}^{+}$ $\left(\mathrm{SF}_{6}\right)_{1}$, which leads to the smallest binding energy among $\mathrm{SF}_{m}^{+}\left(\mathrm{SF}_{6}\right)_{1}$. The inertness of $\mathrm{SF}_{6}$ against $\mathrm{SF}_{m}^{+}$ cations is recognized in two aspects: One is the intrinsically weak intermolecular bond shown in $\mathrm{SF}^{+}\left(\mathrm{SF}_{6}\right)_{1}$; the other is the $\mathrm{F} \cdots \mathrm{F}$ exchange block shown in $\mathrm{SF}_{3}^{+}$ $\left(\mathrm{SF}_{6}\right)_{1}$ and $\mathrm{SF}_{5}^{+}\left(\mathrm{SF}_{6}\right)_{1}$.

\section{Acknowledgments}

We express our appreciation for the financial support of the Morino Foundation for Molecular Science and the grand-in-aid for scientific research from the Ministry of Education, Science, and Culture, Japan. 


\section{References}

1. Jiao, C. Q.; Freiser, B. S. J. Am. Cltem. Soc. 1993, 115, 6268.

2. Creasey, J. C.; Jones, H. M.; Tuckett, R. P.; Hatherly, P. A.; Codling, K.; Powis, I. Chem. Phys. 1993, 174, 441.

3. Becker, H.; Hrusak, J.; Schwarz, H.; Bohme, D. K. J. Chem. Phys. 1994, 100, 1759.

4. Javahery, G.; Becker, H.; Korobov, M. V.; Farber, M.; Cooper, D.; Bohme, D. K. Int. I. Mass Spectrom. Ion Processes 1994, 133, 73.

5. Cheung, Y-S.; Li, W-K.; Chiu, S-W.; Ng, C. Y. I. Chem. Phys. 1994, 101, 3412.

6. Tichy, M.; Javahery, G.; Twiddy, N. D.; Ferguson, E. E. Int. I. Mass Spectrom. Ion Processes 1987, 79, 231.

7. Adams, N. G.; Smith, D.; Tichy, M.; Javahery, G.; Twiddy, N. D.; Ferguson, E. E. J. Chem. Phys. 1989, 91, 4037.

8. Mackay, G. I.; Schiff, H. I.; Bohme, D. K. Int. J. Mass Spectrom. Ion Processes 1992, 117, 387.

9. Latimer, D. R.; Smith, M. A. J. Chem. Phys. 1994, 101, 3410.
10. Kebarle, P. In Techniques for the Study of Ion-Molecule Reactions; Farrar, J. M.; Saunders, W. S., Eds.; Wiley: New York, 1971 ; p 221.

11. Hiraoka, K.; Yamabe, S. In Dynamics of Excited Molecules; Kuchitsu, K., Ed.; Elsevier: Amsterdam, 1994; p 399.

12. (a) Fukui, K. J. Phys. Chem. 1970, 74, 4161; (b) Gonzalez, $C_{\text {.; }}$ Schlegel, H. B. I. Phys. Chem. 1990, 94, 5523.

13. Frisch, M. J.; Head-Gordon, M.; Gill, P. M. E.; Wong, M. W.; Foreman, J. B.; Johnson, B. G.; Schlegel, H. B.; Robb, M. A.; Replogle, E. S.; Gomperts, R.; Andres, J. L.; Raghavachari, K.; Binkley, J. S.; Gonzalez, C.; Martin, R. L.; Fox, D. J.; DeFrees, D. J.; Baker, J.; Stewart, J. J. P.; Pople, J. Gaussian 92, Revision C. Gaussian, Inc., Pittsburgh, P.A, 1992.

14. Rauth, T.; Echt, O.; Mark, T. D. Chem. Phys. Lett. 1993, 201, 345.

15. Walsh, A. D. J. Chem. Soc. 1953, 2260.

16. (a) Berry, R. S. I. Chem. Phys. 1960, 32, 933; (b) Mislow, K. Acc. Chem. Res. 1970, 3, 321.

17. Windas, T. L.; Gordon, M. S.; Davis, L. P.; Burggraf, L. W. 1. An. Chen. Soc. 1994, 116, 3568. 